\title{
Los retos del convenio marco para el control del tabaco en México: un diagnóstico a partir de la Encuesta sobre Tabaquismo en Jóvenes
}

\author{
Raydel Valdés-Salgado, M en C, ${ }^{(1,2)}$ James Thrasher, PhD, ${ }^{(3)}$ Luisa María Sánchez-Zamorano, Dra en C, ${ }^{(1)}$ \\ Eduardo Lazcano-Ponce, Dr en C, (1) Luz Myriam Reynales-Shigematsu, Dr en C, (1) \\ Fernando Meneses-González, M en C, (1) Mauricio Hernández-Ávila, Dr en C. ${ }^{(2)}$
}

\begin{abstract}
Valdés-Salgado R, Thrasher J, Sánchez-Zamorano LM, Lazcano-Ponce E, Reynales-Shigematsu LM, Meneses-González F, Hernández-Avila M. Los retos del convenio marco para el control del tabaco en México: un diagnóstico a partir de la Encuesta sobre Tabaquismo en Jóvenes. Salud Publica Mex 2006;48 supl I:S5-SI6.
\end{abstract}

\section{Resumen}

Objetivo. Analizar la información de la Encuesta sobre Tabaquismo en Jóvenes (ETJ) sobre consumo de cigarrillo y otros indicadores que resulten útiles para una medición inicial de las condiciones en que el país ratificó el Convenio Marco para el Control del Tabaco (CMCT). Material y métodos. La ETJ usa un diseño de muestreo por conglomerado en dos etapas que genera muestras representativas de estudiantes de 12 a 15 años de las escuelas secundarias públicas, privadas y técnicas. Incluye 42024 estudiantes de 492 escuelas en el periodo 2003-2005. Se aplicó un modelo de regresión logística y se obtuvieron razones de momios crudas y ajustadas. Resultados. La prevalencia de fumadores en el mes anterior a la encuesta fue de $19.96 \%$ (IC95\% |8. I-2I.8). El índice de susceptibilidad de iniciarse en el consumo de cigarrillos fue de $25 \%$.A $60 \%$ de los fumadores que compraron cigarrillos no les negaron la venta por ser menores de edad. El $54 \%$ de los estudiantes declara que está expuesto al humo de tabaco en lugares públicos y $86 \%$ ha visto anuncios de cigarrillos en exteriores en el mes previo a la encuesta. Conclusión. Una intervención integral debe orientarse a impedir el acceso de los menores a los cigarri-
Valdés-Salgado R, Thrasher J, Sánchez-Zamorano LM, Lazcano-Ponce E, Reynales-Shigematsu LM,

Meneses-González F, Hernández-Avila M.

Main challenges of the Framework Convention on Tobacco

Control in Mexico:A surveillance report from the

Global Youth Tobacco Survey.

Salud Publica Mex 2006;48 suppl I:S5-SI6.

\section{Abstract}

Objective. This study presents findings from the GlobalYouth Tobacco Survey (GYTS) regarding current cigarette smoking and other tobacco epidemic indicators. Materials and Methods. The GYTS used a two-stage cluster sample survey to generate representative samples of students aged 12 to 15 years old enrolled in public, private and technical schools. The survey was undertaken at 492 schools in 21 cities. The GYTS surveyed 42024 students between the years 2003 and 2005.A logistic regression model was applied and crude and adjusted odds ratios were estimated. Results. The overall current smoking rate during the 30 days preceding the survey was $19.96 \%$ (95\% Cl 18.I-2I.8). Among those who never smoked, 25\% were likely to begin smoking in the following year. Sixty percent of current smokers who bought their cigarettes in stores were not refused purchase because of their age. Exposure to advertising in outside areas was 86 and $54 \%$ of students reported to have been exposed to smoke in public places. Conclusion. Comprehensive tobacco control programs should prevent access by minors to cigarettes, make it more difficult to smoke, and prohibit all forms of tobacco advertising. In addition, options should be provided for those who wish to quit smoking

Este trabajo es parte del proyecto final del primer autor para obtener el título de Master of Public Health (MPH) en la Escuela de Salud Pública de la Universidad Johns Hopkins.

(I) Instituto Nacional de Salud Pública, Cuernavaca, Morelos, México.

(2) The Johns Hopkins University Bloomberg School of Public Health. Department of Epidemiology and the Institute for Global Tobacco Control (IGTC).

(3) Instituto de Investigación en Políticas y Salud. Universidad de Illinois, Chicago, EUA.

Fecha de aprobado: 20 de abril de 2006

Solicitud de sobretiros: Mtro. Raydel Valdés Salgado. The Johns Hopkins University Bloomberg School of Public Health, Department of Epidemiology and the Institute of Global Tobacco Control. 615 N. Wolfe Street, RM. W604I Baltimore, Maryland 2I 205, USA

Correo eléctrónico: rvaldes@jhsph.ed 
llos; dificultar el acto de fumar y prohibir toda forma de publicidad del tabaco.Asimismo, se deben ofrecer opciones a quienes deseen dejar de fumar y educar a los adolescentes y sus familias sobre los daños a la salud de los fumadores y no fumadores.

Palabras clave: tabaco; adolescentes; susceptibilidad de empezar a fumar, México and adolescents and their families should be educated with respect to the dangers of smoking.

Key words: Tobacco; adolescents; smoking susceptibility; Global Youth Tobacco Survey's, Framework Convention on Tobacco Control; Mexico
$\mathbf{E}$ 1 consumo de tabaco es una de las principales causas de muertes evitables y de discapacidad en el mundo. La Organización Mundial de la Salud (OMS) atribuye al tabaco 4.9 millones de muertes anuales y se espera que la cifra exceda los 10 millones para el año 2030. ${ }^{1}$ Entre los rasgos distintivos de la epidemia de tabaquismo se destacan los siguientes: a) es un problema de salud que se ha ido desplazando de los países industrializados a los países en desarrollo; b) la tradicional brecha en el consumo de hombres y mujeres se redujo, y c) por lo general, el consumo se inicia en la adolescencia -cada vez más temprano-, pero el efecto en la morbilidad y mortalidad atribuibles al tabaco se observa a partir de la cuarta década de la vida.

Los tres rasgos anteriores y el peligro para la población que representan han generado respuestas globales para contener este problema de salud pública. Están vigentes dos esfuerzos que se destacan por su magnitud, pero sobre todo por sus objetivos mundiales. El primero de ellos es el Convenio Marco para el Control del Tabaco (CMCT), una iniciativa de la OMS que los estados miembros aprobaron de manera unánime. Como aspecto básico, el CMCT propone una agenda común que coordine esfuerzos en la lucha contra el tabaco, a la vez que ofrece un marco para las medidas de control que habrán de aplicarse en los ámbitos nacional, regional e internacional con el fin de reducir de manera continua y sustancial la prevalencia del consumo y la exposición al humo de tabaco. ${ }^{2}$ México fue el primer país del hemisferio occidental en ratificar el CMCT, el 28 de mayo de 2004.

Junto a un programa de acción común, es necesario disponer de un sistema que evalúe los principales indicadores de consumo y del cumplimiento de las acciones propuestas por el CMCT. En este sentido, el Sistema Global de Vigilancia del Tabaco (SGVT) ${ }^{3}$ representa el segundo esfuerzo antes mencionado. El SGVT fue desarrollado por la OMS, los Centros para el Control y Prevención de Enfermedades de Estados Unidos (CDC, por sus siglas en inglés) y la Asociación Canadiense de Salud Pública. El SGVT apoya a los 192 estados miem- bros de la OMS en la tarea de recoger datos sobre el consumo de tabaco entre adolescentes y adultos. Hasta el momento, el SGVT incluye tres grandes encuestas: la Encuesta Mundial sobre Tabaquismo en Jóvenes, la Encuesta Mundial al Personal de las Escuelas (EMPE) y la Encuesta Mundial a Profesionales de la Salud (EMPS). Las dos primeras ya se aplicaron en México y la tercera está en preparación para aplicarla de inmediato.

En este trabajo se revisa la situación actual de México respecto a los artículos del CMCT, para lo cual los autores se basaron en los resultados de la Encuesta sobre Tabaquismo en Jóvenes (ETJ) que el Instituto Nacional de Salud Pública (INSP) aplicó en 21 ciudades. Los resultados que se presentan a continuación se refieren a áreas criticas para la vigilancia del control del tabaco en México, como prevalencia de consumo (art. 21 del CMCT), humo de tabaco en el ambiente (art. 8), currículum escolar sobre daños causados por el tabaco (art. 12), promoción y publicidad de cigarrillos (art. 13), cesación y dependencia de la nicotina (art. 14), y disponibilidad y acceso de los menores de edad a los productos de tabaco (art. 16).

El objetivo de este trabajo consiste en analizar la información de la ETJ y poner a disposición de las autoridades sanitarias y de todos los involucrados en el control del tabaco en México información actualizada sobre distintos indicadores de la epidemia de tabaquismo, en especial, en lo referido a las etapas más tempranas del consumo de tabaco. Los resultados que se presentan a continuación también pueden usarse como una medición basal de la situación con que México inició sus esfuerzos por implementar las acciones propuestas en el CMCT. Estas mediciones han de resultar de gran utilidad para evaluar los avances en los próximos años.

\section{Material y métodos}

La ETJ recurre a un diseño de muestreo por conglomerado en dos etapas, que genera muestras representativas de estudiantes en los grados asociados con las edades 12 a 15 años. El marco muestral incluye todas 
las escuelas del nivel medio del sector público y privado, así como secundarias técnicas. El listado de escuelas de donde se generó el marco muestral está disponible en el sitio de internet de la Secretaría de Educación Pública (SEP). En la primera etapa del muestreo las escuelas se seleccionan con una probabilidad proporcional a la matrícula de estudiantes en los grados primero, segundo y tercero de secundaria. En la segunda etapa, los salones de clases participantes se seleccionan a partir de una lista de números aleatorios generada para cada escuela, según su matrícula. Todos los estudiantes que asisten a la escuela el día de la aplicación son elegibles para participar si su salón de clases fue seleccionado.

Un factor de ponderación se aplica a cada sujeto participante para ajustar los resultados por las preguntas sin respuestas y las variaciones de la probabilidad de selección por escuela, salón y estudiante. En este artículo, todas las proporciones, diferencias de proporciones y de medias, así como el análisis multivariado mediante un modelo de regresión logística, se realizaron aplicando los pesos correspondientes del factor de ponderación. Las estimaciones se presentan como prevalencias puntuales y como razones de momios crudas y ajustadas con intervalos de confianza (IC) de 95\%. El procesamiento de la información se efectuó con el software STATA 9.1.*

Las principales definiciones que se emplean en este trabajo son fumador, no fumador, nunca fumador (también se refiere como que nunca han fumado) e índice de susceptibilidad de empezar a fumar. Se considera fumador al estudiante que manifestó haber consumido cigarrillos durante los 30 días previos a la encuesta. No fumadores son los estudiantes que no fumaron en el mes previo a la aplicación. Los nunca fumadores son los adolescentes que declaran que nunca probaron un cigarrillo, ni siquiera aspiraron una o dos veces. Los nunca fumadores no susceptibles son los que respondieron Definitivamente no a las preguntas Si uno(a) de tus mejores amigos(as) te ofreciera un cigarrillo, ¿lo fumarías? y ¿Crees que en algún momento durante los próximos 12 meses fumarás un cigarrillo? Aunque en general se menciona el consumo de tabaco, en este artículo tal consumo se refiere en particular a fumar cigarrillos, salvo cuando se menciona en forma explícita "otros productos de tabaco".

La ETJ emplea la misma metodología y un cuestionario común en todos los países participantes. En el caso de México, el cuestionario consta de 76 preguntas y está

\footnotetext{
* Hamilton LC. Statistics with STATA: updated for version 9. 2006 Duxbury, an imprint of Thomson Brooks/Cole, a part of The Thompsom Corporation.
}

disponible en línea en el sitio de internet del Instituto Nacional de Salud Pública (INSP).* Antes de realizar la encuesta, las direcciones de las escuelas participantes informaron a los padres sobre los objetivos de la aplicación que tendría lugar. La aplicación es anónima y no se recogió ningún dato que permita la identificación posterior de los participantes.

En algunos cuadros la información se presenta por zonas geográficas que agrupan más de una ciudad participante, según la siguiente descripción. La primera zona corresponde en sí misma a la Ciudad de México; la zona Norte comprende las ciudades de Ciudad Juárez, Culiacán, Durango, Hermosillo, Monterrey, Nuevo Laredo, Tijuana y Zacatecas. La zona Centro incluye Cuernavaca, León y Toluca. Las ciudades de Guadalajara y Tepic están agrupadas en la zona Occidente, y Puebla y Veracruz, en la zona Oriente. Los resultados de las ciudades de Chetumal, Chilpancingo, Mérida, Oaxaca y Tapachula se agruparon en la zona Sur. El instrumento utilizado es el mismo en todas las ciudades. La información presentada corresponde a los cursos académicos 2003-04 y 2004-05. En México, participaron en la ETJ 42024 estudiantes de 492 escuelas. De ellos, $49.75 \%$ son varones y $50.25 \%$ mujeres, con una edad promedio de 13.4 años (ds 1.23) al momento de la encuesta. Las características de la muestra seleccionada en cada ciudad se presentan en el cuadro I.

El Departamento de Investigación sobre Tabaco del INSP coordinó la ETJ en colaboración con el Consejo Nacional contra las Adicciones (CONADIC) de la Secretaría de Salud, los Consejos Estatales Contra las Adicciones (CECAs) y la Secretaría de Educación Pública en cada una de las ciudades participantes. La Organización Panamericana de la Salud (OPS) y los CDC brindaron asistencia técnica y financiera. Asimismo, el INSP apoyó económicamente y en la logística de la la realización de la encuesta.

\section{Resultados}

En México, el consumo de cigarrillos en la población estudiantil de secundaria fue de $19.96 \%$ (IC95\% 18.1-21.8) en el periodo 2003-2005. El consumo en los 30 días previos a la encuesta fue muy similar entre hombres $20.81 \%$ (IC95\% 19.1-22.5) y mujeres $18.21 \%$ (IC95\% 15.6-20.9); sin embargo, la diferencia es estadísticamente significativa $(p=0.026)$. En el cuadro II se observa que en 10 ciudades participantes no hay diferencias en el consumo de tabaco entre los hombres y las mujeres. En las 11 restantes sí

* www.insp.mx/tabaco/libro/encuesta_tabaco.pdf 
hay diferencias significativas entre las proporciones de fumadores actuales, lo que indica que el consumo entre las mujeres cada vez se asemeja más al de los hombres.

\section{Descripción de proporciones según los artículos del CMCT}

En el cuadro III se presentan estimaciones obtenidas de la ETJ para cada zona que se corresponden con los artículos del Convenio Marco. Este enfoque ya ha sido utilizado para ilustrar el potencial del Sistema Global de Vigilancia del Tabaco. ${ }^{6}$ El artículo 21 del CMCT se refiere a la prevalencia de consumo de cigarrillos y otros productos de tabaco. La situación por zonas refleja que la mitad de los adolescentes ha probado fumar. En este punto, la prevalencia más baja reportada corresponde a la zona Sur (43.4\%), y la más alta a las zonas Centro (54.3\%) y Oriente (53.8\%). Respecto a los

Cuadro I

Características de la muestra seleccionada PARA LA ENCUESTA DE TABAQUISMO EN Jóvenes

\begin{tabular}{|c|c|c|c|c|c|}
\hline & & & $\begin{array}{r}\text { Edad pr } \\
(D\end{array}$ & medio & Prueba $t$ \\
\hline Ciudad & udiantes & Escuelas & Hombres & Mujeres & $p$ \\
\hline I Cd. de México & 2099 & 25 & $13.2(1.21)$ & I3.I (1.09) & 0.835 \\
\hline 2 Cd. Juárez ${ }^{*}$ & 2210 & 25 & $13.1(1.28)$ & $13.1(1.19)$ & 0.938 \\
\hline 3 Cuernavaca $^{*}$ & 2075 & 25 & $13.2(1.15)$ & $13.2(1.06)$ & 0.812 \\
\hline 4 Culiacán $^{\ddagger}$ & 1738 & 22 & $13.3(1.14)$ & $13.3(1.05)$ & 0.992 \\
\hline 5 Chetumal $^{*}$ & 1415 & 9 & $13.3(1.27)$ & $13.3(1.15)$ & 0.381 \\
\hline 6 Chilpancingo & 1888 & 25 & $13.5(1.31)$ & $13.4(1.26)$ & 0.400 \\
\hline 7 Durango & 1970 & 25 & $13.5(1.22)$ & $13.4(1.10)$ & 0.191 \\
\hline 8 Guadalajara $^{*}$ & 2059 & 24 & I3.7 (I.2I) & $13.6(1.08)$ & 0.479 \\
\hline 9 Hermosilloł & 2512 & 25 & $13.3(1.13)$ & $13.3(1.04)$ & 0.593 \\
\hline 10 León ${ }^{\ddagger}$ & 2132 & 25 & $13.5(1.18)$ & $13.4(1.13)$ & 0.834 \\
\hline II Mérida & 1967 & 21 & $13.6(1.31)$ & $13.4(1.18)$ & 0.126 \\
\hline 12 Monterrey ${ }^{\ddagger}$ & 1888 & 25 & $13.4(1.17)$ & $13.4(1.05)$ & 0.758 \\
\hline I3 Nuevo Laredo ${ }^{*}$ & 1416 & 21 & $13.2(1.14)$ & $13.3(1.08)$ & 0.792 \\
\hline 14 Oaxaca* $^{*}$ & 2185 & 24 & $13.2(1.22)$ & $13.1(1.12)$ & 0.584 \\
\hline I5 Puebla* & 1888 & 23 & $13.1(1.21)$ & $13.1(1.17)$ & 0.958 \\
\hline 16 Tapachula $^{*}$ & 2155 & 24 & $13.4(1.38)$ & $13.3(1.28)$ & 0.407 \\
\hline 17 Tepic $^{\ddagger}$ & 2173 & 25 & $13.3(1.13)$ & $13.2(1.07)$ & 0.740 \\
\hline 18 Tijuana* & 2000 & 25 & $13.3(1.14)$ & $13.3(1.01)$ & 0.174 \\
\hline 19 Toluca & 2431 & 25 & $13.3(1.31)$ & $13.2(1.15)$ & 0.203 \\
\hline 20 Veracruz $^{\ddagger}$ & 2267 & 25 & $13.6(1.26)$ & $13.8(1.16)$ & 0.833 \\
\hline 21 Zacatecas ${ }^{\ddagger}$ & 1556 & 24 & $13.3(1.25)$ & I3. $3(1.11)$ & 0.489 \\
\hline Total & 42024 & 492 & $13.3(1.23)$ & $13.3(1.13)$ & 0.596 \\
\hline${ }^{*}$ Encuesta realiz & duran & urso & émico 200 & & \\
\hline ‡ Encuesta realiz & da duran & curso & émico 200 & & \\
\hline
\end{tabular}

fumadores actuales, las zonas Norte y Sur presentan cifras de alrededor de $17 \%$, mientras que en las zonas Centro $(24.7 \%)$ y Oriente $(23.9 \%)$ prácticamente uno de cada cuatro adolescentes fuma. En la Ciudad de México la proporción de fumadores es uno de cada cinco adolescentes. En general, se subestima el uso de otros productos de tabaco, pero entre 5 y $10 \%$ de los adolescentes comunica este tipo de consumo.

El índice de susceptibilidad a iniciarse en el consumo de cigarrillos muestra cifras preocupantes, y así entre 23 y $30 \%$ de los que nunca habían probado el cigarrillo en el momento de la encuesta se mostraba muy dispuesto a hacerlo en los siguientes 12 meses. Otra vez, las cifras más altas proceden de zonas donde se localizan ciudades como Toluca, Puebla, Cuernavaca, León y Veracruz (zonas Centro y Oriente).

Reducir la exposición al humo de tabaco en el ambiente (HTA) es objetivo del artículo 8 del CMCT.

\section{Cuadro II}

Prevalencia de fumadores Por CIUdAd y SEXo

\begin{tabular}{|c|c|c|c|c|c|c|c|c|c|}
\hline \multirow[b]{2}{*}{ Ciudad } & \multicolumn{2}{|c|}{$\begin{array}{c}\text { Prevalencia (\%) } \\
\text { Total }\end{array}$} & \multicolumn{3}{|c|}{$\begin{array}{c}\text { Prevalencia }(\%) \\
\text { Hombres }\end{array}$} & \multicolumn{3}{|c|}{$\begin{array}{c}\text { Prevalencia (\%) } \\
\text { Mujeres }\end{array}$} & \\
\hline & $\%$ & $95 \% / C$ & $n^{*}$ & $\%$ & $95 \% I C$ & $n^{*}$ & $\%$ & $95 \%$ IC & \\
\hline de Méx & 20.2 & $($ (16.3-24.I) & 075 & 20.0 & $(16.7-23.4)$ & 930 & 19.2 & $(13.5-25.0)$ & \\
\hline uár & 22.6 & $(18.7-26.5)$ & 046 & 20.7 & | & 1092 & 23.2 & $(18.1-28.3)$ & 0.20 \\
\hline rnavaca & 20.8 & $(18.2-23.5)$ & 905 & 17.6 & $\mid$ & 1066 & 22.9 & $(18.9-26.9)$ & 0.02 \\
\hline iacán & 10.5 & $(7.20-13.8)$ & 823 & 12.6 & $(8.66-16.7)$ & 855 & 7.3 & $(4.37-10.4)$ & 0.00 \\
\hline etumal & 17.8 & $(14.8-20.9)$ & 636 & 21.5 & $(16.8-26.2)$ & 746 & 14.2 & $(10.9-1)$ & 0.0 \\
\hline panci & 19.4 & $(16.5-22.4)$ & 867 & 23.7 & $(19.3-28.3)$ & 984 & 15.2 & (12.4-1 & 0.00 \\
\hline ango & 22.1 & $(19.3-25.1)$ & 830 & 24.7 & | & 1080 & 19.5 & $(16.9-22.0)$ & 0.09 \\
\hline adalajara & 19.8 & $(15.2-24.5)$ & 926 & 17.9 & | & 1069 & 20.3 & (16.) & 0.14 \\
\hline rmosillo & 20.0 & (16.8 23.3) & 144 & 25.5 & $(1.8-29.3) \mid$ & 292 & 13.8 & $(9.76-17.9)$ & 0.00 \\
\hline & 23.4 & $(20.6-26$ & 043 & 29.7 & $(25.5-33.9)$ & 987 & 17.3 & $(13.7-20.9)$ & 0.00 \\
\hline ida & 19.2 & $(14.2-24.3)$ & 934 & 21.8 & $(14.9-28.7)$ & 992 & 16.7 & (13.0- & 0.01 \\
\hline iterrey & 18.7 & $(15.9-2$ & 951 & 21.9 & (19.5. & 900 & 15.1 & (II.3-1 & 0.00 \\
\hline evo Lar & 16.4 & $(13.3-19.7)$ & 742 & 16.8 & $(12.6-21.0)$ & 633 & 15.2 & $(11.1-19.4)$ & 0.55 \\
\hline xaca & 13.2 & $(\mid 0.7-15.9)$ & 010 & 13.9 & $(10.7-17.2)$ & 1103 & 11.6 & $(8.89-14.5)$ & 0.16 \\
\hline & 25.4 & (19.9- & 903 & 25.2 & (19.3 & 904 & 23.9 & (17.8 & 0.52 \\
\hline pachula & 13.8 & $(10.9-16.7)$ & 046 & 16.3 & $(12.3-20.4)$ & 1055 & 10.8 & $(7.57$ & 0.02 \\
\hline jic & 15.2 & $(13.0-17.5)$ & 991 & 17.3 & (14.53 20.2) & 1119 & 13.0 & $(10.5-15.6)$ & 0.00 \\
\hline Iana & 11.5 & $(9.28-13.7)$ & 981 & 12.4 & $(10.0-14.8)$ & 988 & 10.4 & $(7.69-13.2)$ & 0.15 \\
\hline uca & 27.5 & $(23.5-31.5)$ & 161 & 31.7 & & I 200 & 22.8 & (17. & 0.00 \\
\hline racruz & 18.4 & (15.9 & 000 & 18.8 & 1.6) & I 125 & 16.4 & $(13.3)$ & 0.28 \\
\hline & 20.7 & $(17.8-23.7)$ & 698 & 22.8 & $(19.3-26.5)$ & 794 & 18.6 & $(14.4-22.9)$ & 0.13 \\
\hline & 19.9 & $8.1-21.8)$ & & 20.8 & 22.5) & & 18.2 & $(15.6-20.9)$ & 0.0 \\
\hline
\end{tabular}

$\mathrm{n}^{*}$ Estos valores no suman el total descrito en el Cuadro I pues hay valores missing para las variables Fumador y Sexo 


\section{Cuadro III}

\section{INDICADORES DE LA ETJ" Y SU CORRESPONDENCIA CON LOS ARTÍCULOS DEL CMCT, POR ZONA}

\begin{tabular}{|c|c|c|c|c|c|c|c|}
\hline & $\begin{array}{c}\text { México } \\
\text { 2003-05 }\end{array}$ & Cd. de México & Norte & Occidente & Centro & Oriente & Sur \\
\hline \multicolumn{8}{|c|}{ Artículo 21: prevalencia de consumo de cigarrillos y otros productos de tabaco } \\
\hline \multirow[t]{2}{*}{ Han probado fumar al menos una vez } & 49.8 & 52.9 & 42.1 & 49.5 & 54.3 & 53.8 & 43.4 \\
\hline & $(48.1-51.6)$ & $(49.5-56.5)$ & $(40.1-44.1)$ & $(46.4-52.6)$ & $(52.1-56.5)$ & $(49.2-58.5)$ & $(40.6-46.1)$ \\
\hline \multirow[t]{2}{*}{ Fumador actual (durante el último mes) } & 20.0 & 20.2 & 17.3 & 18.9 & 24.7 & 23.9 & 17.2 \\
\hline & $(|8|-2 \mid .8)$. & $(16.3-24 . I)$ & $(\mid 5.9-18.5)$ & $(15.2-22.6)$ & $(22.6-26.8)$ & $(19.7-28.1)$ & $(14.7-19.7)$ \\
\hline \multirow[t]{2}{*}{ Usa otros productos de tabaco } & 7.6 & 7.4 & 7.7 & 5.9 & 8.4 & 9.4 & 7.2 \\
\hline & $(6.9-8.3)$ & $(6.1-8.8)$ & $(7.0-8.3)$ & $(4.8-7.1)$ & $(7.5-9.2)$ & $(6.9-11.9)$ & $(6.3-8.1)$ \\
\hline No fumadores susceptibles de empezar & 24.9 & 25.1 & 23.1 & 27.1 & 27.2 & 28.5 & 23.1 \\
\hline a fumar & $(23.6-26.3)$ & $(22.1-28.0)$ & $(21.9-24.1)$ & $(24.9-29.3)$ & $(25.2-29.3)$ & $(25.7-31.3)$ & $(21.3-25.0)$ \\
\hline \multicolumn{8}{|c|}{ Artículo 8: exposición al humo de tabaco en el ambiente } \\
\hline \multirow[t]{2}{*}{ Expuestos en el hogar } & 43.9 & 48.8 & 40.4 & 43.2 & 43.2 & 39.8 & 31.2 \\
\hline & $(42.8-45.2)$ & $(46.5-51.1)$ & $(39.1-41.6)$ & $(41.5-44.9)$ & $(41.2-45.2)$ & $(36.6-43.0)$ & $(29.4-32.9)$ \\
\hline \multirow[t]{2}{*}{ Expuestos en lugares públicos } & 53.5 & 56.4 & 51.3 & 57.1 & 53.4 & 48.1 & 44.8 \\
\hline & $(52.3-54.7)$ & $(53.9-58.8)$ & $(50.1-52.5)$ & $(54.8-59.5)$ & $(51.8-55.1)$ & $(45.8-50.4)$ & $(43.046 .6)$ \\
\hline \multirow[t]{2}{*}{ Apoya la prohibición de fumar } & 85.7 & 83.5 & 88.6 & 86.1 & 87.1 & 84.9 & 88.9 \\
\hline & $(84.7-86.6)$ & $(81.5-85.5)$ & $(87.8-89.5)$ & $(84.2-87.9)$ & $(85.7-88.4)$ & $(82.5-87.2)$ & $(87.1-90.6)$ \\
\hline
\end{tabular}

Artículo 12: educación sobre daños a la salud causados por fumar y exposición al humo de tabaco

\begin{tabular}{lccccccc}
$\begin{array}{l}\text { Le han enseñado sobre lo peligroso } \\
\text { de fumar }\end{array}$ & 55.7 & 46.8 & 62.6 & 70.5 & 61.2 & 57.9 & 66.3 \\
& $(53.8-57.6)$ & $(42.7-50.8)$ & $(61.2-64.0)$ & $(66.7-74.4)$ & $(58.4-63.9)$ & $(53.9-61.9)$ & $(64.1-68.4)$ \\
\hline Ha discutido por qué los adolescentes & 32.3 & 27.9 & 34.2 & 42.6 & 34.5 & 34.0 & 38.9 \\
fuman & $(30.8-33.7)$ & $(24.9-30.9)$ & $(32.7-35.6)$ & $(39.2-46.1)$ & $(32.6-36.5)$ & $(31.0-37.1)$ & $(37.4-40.4)$ \\
\hline Le han enseñado sobre los efectos de fumar & 48.3 & 39.3 & 55.6 & 64.2 & 53.5 & 49.5 & 58.8 \\
& $(46.9-49.7)$ & $(36.5-42.0)$ & $(54.2-57.0)$ & $(60.1-68.2)$ & $(51.2-55.8)$ & $(45.6-53.4)$ & $(56.5-61.1)$
\end{tabular}

Artículo I3: publicidad y promoción de los productos de tabaco

$\mathrm{Ha}$ visto actores fumando en TV $\mathrm{y}$ cine

$\begin{array}{lllllll}93.1 & 93.2 & 92.7 & 94.3 & 93.2 & 92.9 & 92.7\end{array}$

\begin{tabular}{lllllll}
$(92.5-93.7)$ & $(92.0-94.4)$ & $(92.1-93.3)$ & $(93.5-95.2)$ & $(92.5-93.8)$ & $(91.2-94.6)$ & $(91.8-93.6)$ \\
\hline
\end{tabular}

\begin{tabular}{|c|c|c|c|c|c|c|c|}
\hline $\begin{array}{l}\text { Ha visto anuncios de cigarrillos en } \\
\text { exteriores }\end{array}$ & $\begin{array}{c}86.0 \\
(84.9-87.2)\end{array}$ & $\begin{array}{c}85.8 \\
(83.4-88.2)\end{array}$ & $\begin{array}{c}86.6 \\
(85.8-87.3)\end{array}$ & $\begin{array}{c}87.9 \\
(86.3-89.6)\end{array}$ & $\begin{array}{c}86.2 \\
(85.3-87.0)\end{array}$ & $\begin{array}{c}85.6 \\
(83.8-87.3)\end{array}$ & $\begin{array}{c}84.3 \\
(83.5-85.1)\end{array}$ \\
\hline Tiene un objeto con el logotipo de & 16.0 & 17.6 & 14.1 & 12.6 & 15.2 & 17.9 & 13.9 \\
\hline una marca de cigarrillos & $(15.0-16.9)$ & $(15.8-19.4)$ & $(\mid 3.2-15.0)$ & (II.I-|4.2) & $(14.0-16.4)$ & $(\mid 4.2-21.7)$ & $(\mid 2.8-15.1)$ \\
\hline
\end{tabular}

Artículo 14: cesación y adicción

\begin{tabular}{|c|c|c|c|c|c|c|c|}
\hline $\begin{array}{l}\text { Fumadores actuales que quieren dejar } \\
\text { el cigarrillo }\end{array}$ & $\begin{array}{c}52.7 \\
(48.0-57.5)\end{array}$ & $\begin{array}{c}52.8 \\
(43.5-62.0)\end{array}$ & $\begin{array}{c}51.8 \\
(48.5-55.2)\end{array}$ & $\begin{array}{c}53.4 \\
(47.9-58.9)\end{array}$ & $\begin{array}{c}57.8 \\
(52.7-62.9)\end{array}$ & $\begin{array}{c}49.0 \\
(40.9-57.2)\end{array}$ & $\begin{array}{c}50.1 \\
43.0-57.3)\end{array}$ \\
\hline Fumadores actuales que ya han & 58.8 & 56.2 & 60.9 & 62.5 & 64.3 & 56.4 & 60.7 \\
\hline intentado dejarlo & $(55.3-62.2)$ & $(49.2-63.2)$ & $(58.2-63.6)$ & $(56.1-68.8)$ & $(60.8-67.7)$ & $(50.7-62.1)$ & $(55.5-65.8)$ \\
\hline Fumadores actuales que han recibido & 66.9 & 66.1 & 65.6 & 64.7 & 69.5 & 70.3 & 70.2 \\
\hline ayuda para dejar de fumar & $(64.2-69.7)$ & $(60.5-71.8)$ & $(62.3-68.8)$ & $(61.4-68.1)$ & $(66.8-72.1)$ & $(65.2-75.5)$ & $(67.0-73.3)$ \\
\hline Fumadores actuales que desean fumar & 5.4 & 5.8 & 5.1 & 5.2 & 5.7 & 4.1 & 5.3 \\
\hline al despertarse & $(3.9-6.9)$ & $(2.9-8.8)$ & $(3.9-6.3)$ & $(2.6-7.8)$ & $(4.2-7.2)$ & $(1.9-6.3)$ & $(3.9-6.6)$ \\
\hline \multicolumn{8}{|l|}{ rtículo 16: acceso de los menores al tabaco } \\
\hline Fumadores que compran cigarrillos & 37.8 & 38.4 & 32.3 & 33.6 & 46.3 & 37.2 & 37.9 \\
\hline en tiendas & $(35.2-40.3)$ & $(33.2-43.7)$ & $(30.4-34.2)$ & $(29.8-37.4)$ & $(43.1-49.4)$ & $(33.6-40.9)$ & $(34.6-4 I .2)$ \\
\hline Fumadores que compraron cigarrillos & 59.6 & 61.8 & 57.8 & 63.6 & 52.6 & 57.2 & 62.8 \\
\hline y su edad no fue problema & $(55.0-64.2)$ & $(53.1-70.6)$ & $(53.3-62.3)$ & $(54.6-72.6)$ & $(47.4-57.8)$ & $(44.7-69.8)$ & $(56.7-68.9)$ \\
\hline La industria tabacalera les ha ofrecido & 11.5 & 11.9 & 10.6 & 9.8 & 13.0 & 12.2 & 10.9 \\
\hline cigarrillos gratis & $(10.3-12.7)$ & $(9.25-14.5)$ & $(9.8-11.3)$ & $(8.1-1 \mid .5)$ & $(I I .9-14.1)$ & $(9.9-14.4)$ & $(9.8 \quad 11.9)$ \\
\hline
\end{tabular}

*Estimado de la proporción de cada indicador, como por ciento, e intervalo de confianza al 95\% 
En este sentido, la ETJ mide la exposición al humo de otros fumadores en el hogar y lugares públicos. La mitad de los estudiantes de la Ciudad de México $(48.8 \%)$ convive con fumadores; en las zonas restantes, la exposición es de alrededor de $40 \%$, pero en la zona Sur menos de uno de cada tres $(31.2 \%)$ está expuesto al humo de tabaco en su casa. La situación de exposición al HTA en lugares públicos es diferente, pues es cercana y aun superior a $50 \%$ en todas las zonas del país. El apoyo a la prohibición de fumar es alto en todas las zonas, ya que más de $83 \%$ piensa que se debería prohibir fumar en lugares públicos.

Hay variaciones por zona en cuanto a los contenidos del plan de estudios acerca del tabaco. Sobre si en el último curso escolar les hablaron de los peligros de fumar, las cifras varían desde menos de $50 \%$ en la capital hasta $70 \%$ en la zona Occidente. En general, una proporción menor respondió que sí recibieron enseñanzas sobre los efectos de fumar, otra vez, con las cifras más bajas en la Ciudad de México (39\%) y las más altas en Occidente (64\%). Las proporciones de quienes discutieron en clase por qué los adolescentes de su edad fuman son aún menores, ya que ningún estimado por zona llega a 40\%.

Es acertado afirmar que la exposición a la publicidad y promoción de los productos del tabaco era intensa en el momento de la encuesta (problema que el art. 13 del CMCT aborda). Un 85\% vio anuncios de cigarrillos en vallas y otros espacios exteriores y casi todos los adolescentes manifiestan que ven actores fumando en cine y televisión (93\%). Además, entre 12 y $18 \%$ cuenta con un objeto promocional con el logotipo de alguna reconocida marca de cigarrillos.

El artículo 14 del CMCT se refiere a la cesación y la adicción a fumar cigarrillos. Según la ETJ, entre los adolescentes fumadores, la mitad quiere dejar el cigarrillo. Esta cifra es bastante consistente en todas las zonas, excepto para la zona Centro, donde la proporción es más alta (58\%). En concordancia con lo anterior, alrededor de $60 \%$ de los fumadores ya hizo algún intento por dejar de fumar en los 12 meses previos a la encuesta, y entre 60 y 70\% recibió algún tipo de ayuda profesional en esa decisión de dejar de fumar. A pesar de su corta edad y de los pocos años que tienen de consumir, alrededor de $5 \%$ de los fumadores muestra indicios de adicción a la nicotina, como es el deseo de fumar de inmediato al despertarse.

El acceso de los menores al tabaco es un punto crítico en un programa de control. No obstante, cifras cercanas a $40 \%$ indican que comprar cigarrillos en las tiendas o en puestos de venta en la calle es la forma más común de obtenerlos. El rango del estimado va desde $32.3 \%$ en el Norte hasta $46.6 \%$ en la zona Centro. Entre los fumadores que compraron cigarrillos, más de la mi- tad responde que no le negaron la venta por tratarse de menores de edad: en la zona Occidente $(63.6 \%)$, en la Sur $(62.8 \%)$ y en la Ciudad de México (61.8\%). Uno de los indicadores con menos variabilidad entre las ciudades participantes es que alrededor de $11 \%$ de los encuestados consigna que representantes de la industria tabacalera les ofrecieron muestras de cigarrillos gratis. Todo lo anterior evidencia los retos para cumplir con lo que establece el artículo 16 del CMCT.

\section{Análisis bivariado}

A continuación se presentan las razones de momios crudas -crude odds ratio $\left(O R_{c}\right)$ - respecto a la condición de fumador para diferentes características de los estudiantes de su entorno, así como actitudes y creencias.

Los adolescentes que reconocen haber consumido otros productos de tabaco tienen 2.7 veces más probabilidades de también ser fumadores de cigarrillos $\left(O R_{c}=2.7 ; I C 95 \%\right.$ 2.3-3.2). La disponibilidad de dinero para gastar guarda un relación positiva con el consumo de cigarrillos, para ser entre cinco y seis veces superior respecto de los que carecen de capacidad económica para comprarlos. Por ejemplo, los que cuentan con 50-100 pesos al mes tienen casi seis veces más probabilidades de ser fumadores $\left(O R_{c}=5.6\right.$; IC95\% 4.86.5) y siguen los que disponen de más de 500 pesos $\left(O R_{c}=5.4 ; I C 95 \%\right.$ 4.2-6.8).

Convivir con fumadores también incrementa las probabilidades de ser fumador. Cuando fuman los dos padres (o tutores) las probabilidades se duplican $\left(O R_{c}=1.9\right.$; IC95\% 1.5-2.5), y la influencia de la figura materna fumadora parece ser mayor $\left(O R_{c}=2.2 ; I C 95 \% 1.7-2.8\right)$ que la de la figura paterna $\left(O R_{c}=1.6\right.$; IC95\% 1.3-1.9). Los adolescentes a quienes nadie de su familia les habló de los efectos dañinos del cigarrillo están expuestos 1.7 veces más a fumar (IC95\% 1.5-1.9).

En cuanto a si fumar es un comportamiento que facilita hacer más amistades, las percepciones varían según si se refieren a los o las adolescentes fumadores. Los que consideran que las adolescentes que fuman consiguen más amistades tienen $20 \%$ más de probabilidades de convertirse en fumadores $(O R=1.2$; IC $95 \%$ 1.1-1.4). En cambio, quienes creen que los adolescentes que fuman gozan de más amistades tienen $46 \%$ menos probabilidades de ser fumadores $\left(O R_{c}=0.54\right.$; IC $95 \% 0.46$ $0.63)$. El atractivo percibido en los fumadores es otra asociación cruda significativa. Por ejemplo, quienes piensan que fumar determina que las adolescentes luzcan menos atractivas tienen $41 \%$ menos probabilidades de ser fumadores $\left(O R_{c}=0.59\right.$; IC95\% 0.51-0.69); algo similar, pero más acentuado, ocurre con los que ven a los fumadores varones como menos atractivos $\left(O R_{c}=0.31\right.$; 
IC95\% 0.26-0.35). De igual modo, las probabilidades de ser fumador son $64 \%$ más bajas entre los que opinan que fumar hace a las personas sentirse menos cómodas en las fiestas $\left(O R_{c}=0.36\right.$; IC95\% 0.30-0.43).

Los que tienen pensamientos positivos cuando ven a una mujer fumadora, como que es popular, inteligente, o más sexy, tienen nueve veces más probabilidades de ser fumadores que los que piensan que una mujer que fuma es alguien a quien le falta confianza, o está nerviosa, o no es inteligente $\left(O R_{c}=9.3\right.$; IC95\% 3.2-27.1). Esa asociación también se verifica respecto a los que tienen pensamientos positivos cuando ven a un hombre fumador $O R_{c}=3.5$; $(I C 95 \%$ 2.7-4.5). Los que piensan que fumar por uno o dos años y después dejar el cigarrillo no entraña riesgos para la salud tienen casi cuatro veces más probabilidades de volverse fumadores $\left(O R_{c}=3.6\right.$; IC95\% 3.1-4.2).

Los que abrigan dudas o estiman que no es dañino fumar se enfrentan a una probabilidad tres veces mayor de ser fumadores que aquellos que están convencidos de que el fumar es dañino $\left(O R_{c}=3.1\right.$; IC95\% 2.6 a 3.7). Comparados con los que no tienen amigos que fumen, aquellos con algunos o la mayoría de sus amigos fumadores se exponen al doble de probabilidades de ser fumadores $\left(O R_{c}=2.0 ;\right.$ IC95\% 1.8-2.3) y cuando todos los amigos fuman, las probabilidades de convertirse en uno más son mayores de tres veces $\left(O R_{c}=3.2 ; I C 95 \%\right.$ 2.7-3.8). Los fumadores son quienes mejor conocen lo difícil que resulta dejar de fumar una vez que se empieza, pues entre ellos hay $84 \%$ más probabilidad de recibir una respuesta afirmativa a esa pregunta respecto a los no fumadores $\left(O R_{c}\right.$ = 1.8; IC95\% 1.6-2.1). Es preocupante que algunos adolescentes piensen que es seguro fumar por uno o dos años y después dejarlo, pues quienes sostienen estas ideas tienen casi $80 \%$ más probabilidades de resultar fumadores $\left(O R_{c}=1.8 ;\right.$ IC $95 \%$ 1.7-1.9).

Respecto al humo de los otros fumadores, los que no creen que les hace daño tienen $82 \%$ más probabilidades de ser fumadores $\left(O R_{c}=1.8 ; I C 95 \% 1.5-2.2\right)$. Los que están expuestos al humo de tabaco de segunda mano (HTSM) de su casa tienen dos y media veces más probabilidades de fumar $\left(O R_{c}=2.5\right.$; IC95\% 2.2-2.8) y la exposición al HTSM en lugares públicos también se asocia con ser fumador $\left(O R_{c}=3.1\right.$; IC95\% 2.2 a 2.8). No es sorprendente que quienes apoyan que se debe prohibir fumar en lugares públicos tengan $83 \%$ menos probabilidades de ser fumadores $\left(O R_{c}=0.17 ;\right.$ IC95\% 0.15-0.19). Una situación similar surge entre los que están a favor de que se prohíba fumar en las discotecas $\left(O R_{c}=0.22 ;\right.$ IC $\left.95 \% 0.20-0.30\right)$.

Parece que los fumadores son más perceptivos a los mensajes en contra del tabaco que aparecen en los medios de comunicación que los no fumadores, pues se observa una relación inversa entre los que manifies- tan que han visto pocos $\left(O R_{c}=0.89 ;\right.$ IC $\left.95 \% 0.81-0.97\right) \mathrm{o}$ ninguno de éstos $\left(O R_{c}=0.81 ;\right.$ IC95\% 0.69-0.93). Detentar algún objeto o prenda de vestir con el logotipo de una marca de cigarrillos es 2.6 veces más probable entre los fumadores $\left(O R_{c}=2.6\right.$; IC95\% 2.3 a 2.9). Asimismo, son los fumadores quienes más refieren que representantes de la industria tabacalera les han ofrecido cigarrillos gratis $\left(O R_{c}=1.9\right.$; IC95\% 1.6-2.2).

Sobre lo que les enseñan en las escuelas acerca del tabaquismo también se observan diferencias, pues aquellos que refieren pláticas sobre los peligros de fumar en cualquiera de sus materias tienen 13\% menos probabilidades de volverse fumadores $\left(O R_{c}=0.87\right.$; IC95\% 0.760.99). Una asociación similar (17\% menos) se observa entre los que manifiestan haber discutido en clases por qué fuman los adolescentes $\left(O R_{c}=0.82\right.$; IC95\% 0.73-0.92).

El cuestionario de México incluye una sección que explora las percepciones sobre la industria tabacalera. Por ejemplo, respecto a los no fumadores, los que fuman afirman estar en absoluto desacuerdo con que se prohíba la producción de cigarrillos en forma definitiva $\left(O R_{c}=3.1\right.$; IC95\% 2.8-3.5). Los que consideran que la culpa que se hace recaer en las tabacaleras de que los jóvenes fumen es demasiado alta tienen $16 \%$ menos probabilidades de ser fumadores $\left(O R_{c}=0.84 ;\right.$ IC95\% 0.77-0.93). Los fumadores tienen más probabilidades de pensar que las tabacaleras no esconden los daños que causa fumar $\left(O R_{c}=\right.$ 1.6; IC95\% 1.4-1.8) y que los anuncios de cigarrillos no son menos sinceros que los de otros productos $\left(O R_{c}=\right.$ 1.3; IC95\% 1.1-21.5). En el mismo sentido, los fumadores son quienes más convencimiento muestran respecto de que las tabacaleras sí se preocupan por la salud de quienes consumen su producto $\left(O R_{c}=1.6\right.$; IC95\% 1.4-1.8) y que dicha industria no esconde que los cigarrillos son adictivos $\left(O R_{c}=1.4 ; I C 95 \%\right.$ 1.3-1.6).

Dos preguntas que la Secretaría de Educación Pública distinguen con claridad a los fumadores de los no fumadores. Una de ellas inquiere sobre si consideran que el tabaco es una droga. Los que responden a la misma que no saben tienen el doble de probabilidades de convertirse en fumadores $\left(O R_{c}=2.2 ; I C 95 \% 1.8-2.7\right)$, mientras que entre los que responden que no las probabilidades superan el triple $\left(O R_{c}=3.4 ;\right.$ IC $95 \%$ 2.9-3.9). La otra pregunta se refiere a si se considera muy joven para fumar y, otra vez, los que responden que no saben tienen más del doble de probabilidades de volverse fumadores $\left(O R_{c}=2.4 ; I C 95 \% 1.9-2.9\right)$ y los que responden que no, tienen cuatro veces más probabilidades de serlo $\left(O R_{c}=3.9\right.$; IC $95 \%$ 3.3-4.7).

Entre las características sociodemográficas, por cada año de edad las probabilidades de ser fumador se incrementan $44 \%$ respecto a la edad anterior $\left(O R_{c}=1.4 ;\right.$ IC $95 \%$ $1.4-1.5)$. En general, las mujeres exhiben $15 \%$ menos pro- 
babilidades de ser fumadoras si se comparan con los varones $\left(O R_{c}=0.85 ;\right.$ IC95\% 0.73-0.98). El hecho de vivir en una zona u otra de México no incrementa las probabilidades de ser fumador, comparadas con las de los adolescentes que viven en la capital del país.

\section{Análisis multivariado}

En el cuadro IV se presenta el modelo de regresión logística que incluye los principales factores asociados con el consumo de tabaco durante los 30 días previos a la aplicación de la ETJ. Los factores se agruparon según su relación con distintas medidas de control propuestas por los artículos del Convenio Marco para el Control del Tabaco. Se presentan las razones de momios ajustadas $\left(O R_{\mathrm{aj}}\right)$ por cada uno de los factores presentes en el modelo y se incluyeron también las razones de momios crudas $\left(O R_{c}\right)$, que antes se describieron en detalle. Este modelo se obtuvo agregando una por una las variables a considerar; aquéllas cuya contribución no fue significativa se excluyeron. La variable sexual se consideró dentro del modelo, aunque carece de significancia estadística. El modelo que se describe a continuación es poco parsimonioso, pero incluye todos los factores estadísticamente significativos susceptibles de modificar con intervenciones que apliquen las medidas que el Convenio Marco propone.

Como variable continua, la edad medida muestra un incremento de $37 \%$ en las probabilidades de ser fumador, después de ajustar por las otras variables en el modelo $\left(O R_{\mathrm{ajj}}=1.37 ;\right.$ IC95\% 1.32-1.42). Ya se mencionó que la probabilidad menor de $5 \%$ de ser fumador entre las mujeres no es significativa, porque el intervalo de confianza a $95 \%$ incluye la hipótesis nula de falta de diferencia entre los sexos $\left(O R_{\mathrm{aj}}=0.95 ; I C 95 \%\right.$ 0.76-1.19). Cuando algunos, o la mayoría, de los amigos o amigas fuman se incrementan las probabilidades de ser fumador en $54 \%$ $\left(O R_{\mathrm{aji}}=1.54 ;\right.$ IC95\% 1.26-1.88). En cambio, cuando todas las amistades fuman, las probabilidades de ser fumador son $87 \%$ superiores respecto a los que no tienen amigos fumadores $\left(O R_{\mathrm{aj}}=1.87\right.$; IC $95 \% 1.52$ a 2.32).

En relación con quienes manifestaron que no disponían de dinero, cada incremento de 50 pesos mexicanos se vincula con un aumento de $30 \%$ en las probabilidades de volverse fumador. Para ser estrictos, esta relación lineal se observa hasta los que disponen de $\$ 150$ mensuales $\left(O R_{\mathrm{aj}}=1.30 ; I C 95 \%\right.$ 1.20-1.40). Entre los que disponen de más de 150 pesos al mes (las categorías consideradas son 250, 500, 1000 y más de mil pesos mensuales) , se observa una disminución de $34 \%$ en las probabilidades de ser fumador $\left(O R_{\mathrm{aj}}=0.66 ; I C 95 \% 0.56-0.77\right)$. Hasta donde se conoce, es ésta la primera vez que se reconoce una relación inversa entre nivel socioeconómico alto y con- sumo de tabaco en México, lo cual no constituye una sorpresa porque en otras encuestas el extremo superior del nivel socioeconómico no se incluye.

La exposición al humo de tabaco de segunda mano (HTSM) aparece de nuevo asociada a la condición de fumador y así, entre los expuestos al HTSM en el hogar, las probabilidades de convertirse en fumador son $33 \%$ mayores respecto a los que no conviven con fumadores $\left(O R_{\mathrm{aj}}=1.33\right.$; IC95\% 1.12-1.57). Los que consignaron haber estado expuestos al HTSM en lugares públicos tienen 2.2 veces más probabilidades de ser fumadores $\left(O R_{\mathrm{aj}}=2.16\right.$; IC95\% 1.80-2.58).

También se encontraron creencias que distinguen de manera marcada a fumadores de no fumadores y que aparecen como factores protectores o de riesgo a tomar en cuenta. Por ejemplo, los que consideran que el tabaco no es una droga tienen $77 \%$ más probabilidades de ser fumadores respecto a los que consideran al tabaco una droga $\left(O R_{\mathrm{aj}}=1.77\right.$; IC95\% 1.55-2.02). En el mismo sentido, considerar que se es muy joven para fumar actúa como un factor protector, y quienes no lo piensan así tienen $45 \%$ más probabilidades de fumar $\left(O R_{\mathrm{aj}}=1.45\right.$; IC95\% 1.23-1.72). De igual manera, aquellos que piensan que fumar cigarrillos no es dañino para su salud tienen casi el doble de probabilidades de convertirse en fumadores $\left(O R_{\mathrm{aj}}=1.99\right.$; IC95\% 1.58-2.53).

El desconocimiento de la adicción y de las consecuencias futuras se aprecian en las respuestas a las preguntas de si creen que es difícil dejar de fumar una vez que se empieza y si creen que es seguro para la salud fumar sólo por uno o dos años y después dejar el cigarrillo. En la primera de ellas llama la atención que son los fumadores los que principalmente responden de manera afirmativa $\left(O R_{\mathrm{aj}}=1.32 ; I C 95 \% 1.11-1.57\right)$. Respecto a si es seguro fumar por uno o dos años, quienes así lo estiman se exponen $42 \%$ más a ser fumadores $\left(O R_{\mathrm{aj}}=1.42 ; I C 95 \% 1.30\right.$ a 1.55).

Para finalizar, también se halló que la posesión de prendas u objetos con el logotipo de marcas de cigarrillos está asociada con ser fumador $\left(O R_{\mathrm{aj}}=1.58 ; I C 95 \%\right.$ 1.35-1.86) y que la industria tabacalera se promueve en forma activa entre los adolescentes regalándoles cigarrillos $\left(O R_{\mathrm{aj}}=1.32\right.$; IC95\% 1.03-1.69).

\section{Discusión}

En México, 20\% de los estudiantes de secundaria son fumadores. La ETJ aplicada de manera consecutiva durante los cursos académicos 2003-04 y 2004-05 en 21 ciudades ofrece estimaciones valiosas de la situación con que el país inició sus esfuerzos por implementar las medidas propuestas en el CMCT. 


\section{Cuadro IV \\ Factores asociados a haber fumado CIGARROS DURANTE EL MES PREVIO A LA ENCUESTA Y SU RELACIÓN con los artículos del Convenio Marco para el Control del Tabaco}

Factores

OR $O R_{\text {ajustado }} \quad I C 95 \%$ para $O R_{\text {adj }}$

Características sociodemográficas asociadas a la prevalencia de consumo de cigarros

Edad (variable continua)

Sexo

Hombre

$\begin{array}{llll}1.44 & 1.37 & 1.32 & 1.42\end{array}$

Mujer

$1.00 \quad 1.00$

$\begin{array}{llll}0.85 & 0.95 & 0.76 & 1.19\end{array}$

¿Alguno de tus mejores amigos o amigas fuma?

Ninguno de ellos $\quad 1.00 \quad 1.00$

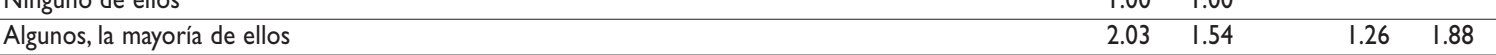

Todos fuman

$\begin{array}{llll}3.23 & 1.87 & 1.52 & 2.32\end{array}$

Factores sensibles a lo propuesto en el artículo 6: Aplicación de impuestos

En un mes, ¿de cuánto dinero dispones para gastar?

No dispongo de dinero

$1.00 \quad 1.00$

Por cada $\$ 50$ pesos de incremento, hasta $\$ 150$

$1.17 \quad 1.30$

$\mathrm{I} .20 \quad \mathrm{I} .40$

Nivel socioeconómico alto

Dispone de menos de $\$ 150$ al mes

$1.00 \quad 1.00$

Disponen de $\$ 250, \$ 500$ y $\$ 1000$ o más al mes

$\begin{array}{llll}1.20 & 0.66 & 0.56 & 0.77\end{array}$

Factores sensibles a lo propuesto en el artículo 8: Reducción de la exposición al humo de tabaco

Durante la semana previa, ifumo alguien en tu presencia estando en tu casa?

$\begin{array}{lrrr}\text { No } & 1.00 & 1.00 & 2.46 \\ \text { Sí } & 1.33 & 1.12 & 1.57\end{array}$

Durante la semana previa, ¿fumo alguien en tu presencia estando fuera de tu casa?

$\begin{array}{lrrr}\text { No } & 1.00 & 1.00 & 3.14 \\ \text { Sí } & 2.16 & 1.80 & 2.58\end{array}$

Factores sensibles a lo propuesto en los artículos 12 y 14 : Educación sobre daños y adicción

¿Consideras que el tabaco es una droga?

Sí

$\mathrm{N}$

$1.00 \quad 1.00$

$\begin{array}{llll}2.84 & 1.77 & 1.55 & 2.02\end{array}$

¿Consideras que eres muy joven para fumar?

$\begin{array}{lll}\text { Sí } & 1.00 & 1.00\end{array}$

$\begin{array}{lll}\text { No } & 3.18 & 1.45\end{array}$

¿Crees que fumar cigarros es dañino para tu salud?

$\begin{array}{lll}\text { Sí } & 1.00 \quad 1.00\end{array}$

$\begin{array}{lllll}\text { No } & 3.12 & 1.99 & 1.58 & 2.53\end{array}$

¿Crees que es seguro para tu salud fumar por uno o dos años, siempre que después lo dejes?
No
$1.00 \quad 1.00$

Sí

$\begin{array}{llll}1.78 & 1.42 & 1.30 & 1.55\end{array}$

¿Crees que es difícil dejar de fumar una vez que se empieza? No $1.00 \quad 1.00$

$\begin{array}{llll}1.84 & 1.32 & 1.11 & 1.57\end{array}$

Factores sensibles a lo propuesto en el artículo 13: Prohibición de la promoción y publicidad

¿Tienes alguna prenda u objeto con en logotipo de una marca de cigarros?

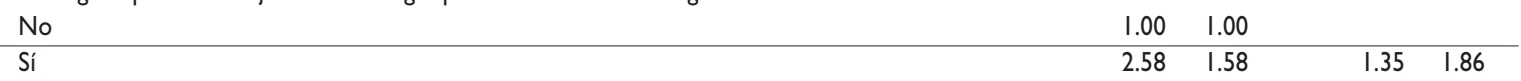

Factores sensibles a lo propuesto en el artículo 16: Restricción del acceso de los menores al tabaco

¿Alguna persona de la industria tabacalera te ha ofrecido cigarros gratis?

$\begin{array}{llll}\text { No } & 1.00 & 1.00 & 1.00 \\ \text { Sí } & 1.32 & 1.03 & 1.69\end{array}$


Quizá el rasgo más distintivo de la epidemia de tabaquismo en México es que a las edades de inicio del consumo de cigarrillos, las prevalencias de mujeres y hombres son similares en las distintas regiones del país. El hecho de que se encuentren en desaparición las diferencias de género entre quienes fuman durante la adolescencia está documentado como un patrón emergente en el mundo. ${ }^{5}$ Numerosos estudios prueban que el riesgo de que una persona empiece a fumar guarda estrecha relación con la exposición a la publicidad de cigarrillos, y en este sentido, la venta de tabaco a las mujeres jóvenes representa hoy la mayor oportunidad publicitaria para un producto a escala mundial. ${ }^{5}$ La publicidad y las diversas formas de mercadeo que asocian al cigarrillo con independencia, glamour, imagen corporal y romance apuntan específicamente a la mujer pero no se detienen aquí; su mentor, la industria tabacalera, penetró el mercado femenino patrocinando eventos de moda y deportivos para la mujer con gran éxito comercial. ${ }^{6}$

Los datos derivados de la ETJ tienen grandes implicaciones para la acción. En primer lugar, muestran una realidad que en cierta forma ya era conocida: los adolescentes fuman desde edades tan tempranas como los 11 o 12 años e, incluso, desde antes. La mitad de ellos ha probado el cigarrillo. A pesar de las leyes que imperan, no reviste mayor dificultad para los menores acceder a los cigarrillos; la exposición al humo de tabaco en el ambiente está lejos de representar una situación restringida. De igual modo, no sorprende confirmar que la publicidad exterior, los medios de comunicación y otros recursos indirectos continúen promoviendo el cigarrillo como producto de consumo y el acto de fumar como conducta socialmente aceptada.

Pese a ello, no se sabía que $25 \%$ de los estudiantes que nunca fumó era susceptible a empezar a fumar en los 12 meses siguientes a la encuesta. La susceptibilidad de empezar a fumar es un indicador que ha sido validado como fuerte predictor de inicio del consumo de cigarrillos entre los adolescentes. ${ }^{7}$ Tampoco estaba documentado que a $11 \%$ de ellos la industria tabacalera les ofrece cigarrillos gratis. Estos datos reflejan cuánto resta por hacer para que el espíritu del CMCT tenga plena vigencia en México.

Así como la realidad que refleja la ETJ no era del todo desconocida, las medidas propuestas en el CMCT tampoco son algo a lo que se accede por primera vez. Tomar los datos que aquí se presentan como una medición basal, como una situación que debe superarse en forma sustantiva, es lo que le daría sentido a repetir el esfuerzo enorme que significa aplicar la ETJ en un país grande como México. La aplicación futura de esta encuesta no se justificaría, ni sus resultados interesarían si lo que aquí se presenta no mueve a la acción ni genera el compromiso de modificar estos datos para bien. Si las medidas del CMCT no se aplican tal y como señala ese documento, y aun en forma más estricta, sería ingenuo pensar que la situación hoy descrita va a cambiar, o lo será para mal, si se continúa cediendo al empuje de la siempre voraz y poderosa industria tabacalera.

Es evidente que si se interviene sobre los factores presentados en el modelo aquí descrito la situación puede cambiar. Se necesita más educación sobre los daños que causa el tabaco a la salud una o dos décadas después de haber empezado a fumar, más educación sobre la adicción a la nicotina y más educación sobre los trucos publicitarios que con suma facilidad confunden las mentes jóvenes. ¿Se trata pues de esperarlo todo de las escuelas? Por supuesto que no. En primer lugar porque la Encuesta Mundial al Personal de las Escuelas (aplicada en México en 2003) comprobó la falta de recursos para hacerlo y lo poco preparados que están los maestros para emprender esta tarea. ${ }^{8}$ ¿Cómo transmitir entonces que el cigarrillo es dañino si la publicidad omnipresente afirma lo contrario? ¿Cómo desincentivar el consumo de un producto que está en todas partes y que se consigue en forma fácil y a precios accesibles? ¿Si el humo de otros afecta tanto, por qué hay tanta libertad para fumar en los lugares públicos? No es sólo con mensajes bienintencionados que se cambia el panorama aquí descrito.

Capacidad nacional para controlar el tabaco se define como la capacidad de cada país para desarrollar y emprender acciones multisectoriales e integrales que faciliten las estrategias adecuadas de prevención y control que reduzcan el consumo de tabaco. ${ }^{9}$ Lo anterior se refleja en tres componentes esenciales en cualquier escenario nacional: a) conocimientos y liderazgo, b) infraestructura y c) evidencia empírica. En el caso de México -de los primeros en ratificar el CMCT y con numerosas instituciones científicas y profesionales dedicadas a este tema-, el primer punto no es un problema. También se cuenta con la infraestructura necesaria, y prueba de ello es la realización misma de la ETJ con la colaboración de instituciones nacionales y estatales. Para concluir, el sistema de encuestas nacionales, a las cuales se suman las tres encuestas que constituyen el Sistema Global de Vigilancia del Tabaco (SGVT), garantiza la información actualizada a que se alude cuando se establece la necesidad de evidencia empírica. Sin embargo, los resultados de la ETJ reflejan que la articulación de estos tres componentes todavía está lejos de tener control sobre el problema del tabaco.

¿Dónde intervenir y cómo medir si se está avanzando? El cuadro III de este trabajo ofrece una matriz 
bastante completa de los indicadores que deberían cambiar si las acciones implementadas son efectivas. No obstante, quizá tome algún tiempo lograr cambiarlos todos y por eso hay que pensar en seleccionar algunos, los que sean más urgentes y sensibles a las intervenciones directas.

La figura 1 retoma algunas medidas ya conocidas y las reordena creando tres barreras y un canal de salida como modelo de intervención. Las tres barreras están orientadas a impedir el acceso al producto, dificultar la ejecución de la conducta y debilitar una percepción errónea. El canal de salida tiene un diseño que favorece y facilita dejar de fumar. Los elementos incluidos en este esquema se identificaron de los resultados de la ETJ y aparecen como covariables significativas en el modelo de los factores asociados a fumar durante los 30 días previos a la encuesta presentado en este trabajo.

La barrera 1 se implementaría aplicando más impuestos al precio de los cigarrillos, haciendo cumplir la prohibición de venta a menores y castigando la distribución de cigarrillos gratis. Esta barrera para impedir el acceso a los cigarrillos se sostiene en lo establecido en los artículos 6 y 16 del CMCT. La barrera 2, para dificultar el acto de fumar, quedaría establecida con la entrada en vigor de los espacios libres de humo en los lugares de trabajo y de recreación y, claro está, vigilando el cumplimiento de esta medida. Esta segunda barrera concretaría lo que sugiere el artículo 8 del CMCT. Para establecer la barrera 3 se debe prohibir toda forma de publicidad y promoción de los productos de tabaco y así debilitar la percepción de que fumar es

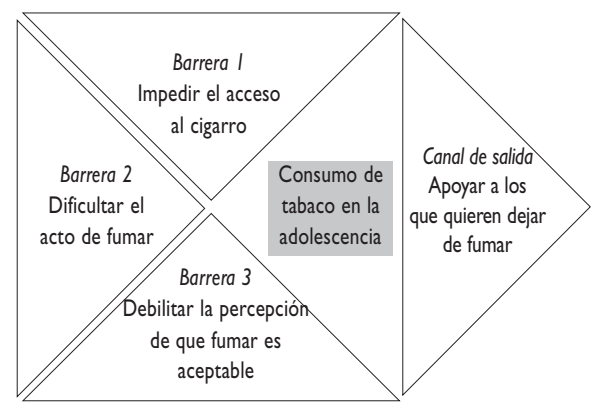

Mensajes a los adolescentes:

- El tabaco es una droga

- La nicotina es adictiva

- Se puede rechazar el cigarro

Figura I. Propuesta de intervención basada en los Resultados de la ENCUESTA sobre Tabaquismo en Jóvenes y en el Convenio Marco para el Control del TABACO normativo y socialmente aceptable. La ETJ revela que la mitad de los adolescentes fumadores quiere dejar el cigarrillo y que $60 \%$ de ellos ya hizo algún intento por dejarlo. Entonces hay que implementar un canal de salida para identificar y ofrecer opciones a esos fumadores, lo cual no es otra cosa que lo planteado en el artículo 16 del CMCT.

A pesar de que el modelo estadístico que acaba de presentarse pareciera sugerir de manera exclusiva intervenciones sobre los factores que se midieron a nivel individual, los autores proponen un esfuerzo integral. Está bien establecido que las intervenciones que derivan del compromiso político con el control del tabaco, que pone en juego toda la infraestructura disponible, así como que toman en cuenta la etapa de la epidemia del tabaquismo en que se encuentra cada país, tienen más probabilidades de éxito que las intervenciones implementadas en un contexto individual. ${ }^{10}$ Por lo tanto, esta propuesta de intervención consiste en crear barreras que alejen a los jóvenes del tabaco.

Mas allá del ordenamiento gráfico que se le asigne a estos factores, el mensaje de la ETJ es que la disponibilidad de dinero es un aspecto importante para poder fumar y por lo tanto sensible de atacar con impuestos. La alta exposición al HTSM indica que existen demasiadas facilidades para poder fumar y que se debe revertir este panorama. Además, la industria mantiene una promoción activa de su producto y estimula la percepción de que fumar es normativo. Aunque conseguir cigarrillos sigue siendo bastante fácil para los menores de edad, la mitad de los adolescentes fumadores desea dejar el cigarrillo y por ello hay que concentrarse en ayudarlos. Este conjunto de acciones debe tener como trasfondo una campaña educativa enfocada a mensajes muy puntuales: el tabaco es una droga; el tabaco contiene una sustancia adictiva que dificulta dejar de fumar y sí es posible rechazar el cigarrillo que ofrece un amigo. Estos tres últimos elementos se retomaron del modelo aquí descrito y su inclusión en la figura 1 está en concordancia con el artículo 12 del CMCT.

La presentación de resultados por ciudades y por regiones ofrece una oportunidad para revisar la efectividad de los programas de control del tabaco en cada lugar. La sugerencia de enfocar los esfuerzos hacia las tres barreras aquí propuestas puede ser una experiencia a iniciar en el contexto estatal y, después de evaluar su impacto y corregir su implementación, se podría extender a la región, para finalizar aplicándola a nivel nacional. Experiencias en otros países muestran una relación causal entre los cambios introducidos en programas locales de control del tabaco y la disminución en el consumo per cápita de cigarrillos. ${ }^{11}$ 
Un resultado que merece especial atención dentro de lo que nos muestra la ETJ es que por primera vez se documenta para México que las probabilidades de ser fumadores son más bajas en los estratos socioeconómicos altos, comparados con los adolescentes que disponen de menos de 150 pesos mensuales. Si ello obedece a pertenecer a familias con mayor educación y por tanto más preocupadas por la salud, requiere una investigación más detallada. Lo cierto es que los mensajes educativos que se requieren dentro de un esfuerzo integral para el control del tabaco no pueden recaer solamente en las acciones que el gobierno emprenda o en la educación que brinden las escuelas. La familia, como entorno inmediato y cotidiano y como proveedora de recursos, debe participar en forma activa. En este contexto, habría que diseñar mensajes no sólo para los adolescentes sino también para los padres.

Mas allá de su utilidad para evaluar el efecto de las medidas de control que se implementen, la ETJ encierra un valor diagnóstico inmediato. En este sentido, el consumo equiparable de hombres y mujeres, así como la alta susceptibilidad de empezar a fumar entre los que nunca lo hicieron alertan sobre cualquier estimado de morbilidad y mortalidad atribuible al tabaco elaborado en el pasado ya que con seguridad, al no tomar en cuenta estos dos rasgos, subvalora la verdadera magnitud del problema por enfrentar. Esta preocupación ya se expresó de manera global, derivada también del análisis de la ETJ en otras regiones del mundo. ${ }^{12}$

Los resultados obtenidos de la ETJ están sujetos al menos a tres limitaciones. Primero, es una muestra tomada de entre los adolescentes que asisten a la escuela. Segundo, los datos se obtuvieron de los estudiantes que asistieron a la escuela el día de la aplicación y cuyos salones de clase fueron seleccionados para participar. Tercero, los datos se basan en el autorreporte, por lo que los estudiantes pueden haber subvalorado o sobrevalorado las respuestas sobre sus conductas y creencias. Por otro lado, las fortalezas de estos resultados son fruto del uso de una metodología común y del mismo instrumento; del riguroso proceso de muestreo de las escuelas y salones, así como del uso de los ponderadores derivados de ese diseño que se aplicaron en cada una de las estimaciones aquí presentadas. Otros dos puntos a destacar son la inclusión de escuelas públicas, privadas y secundarias técnicas, así como de todos los estratos socioeconómicos.

En este artículo se presenta la situación con que México está enfrentando la epidemia de tabaquismo y en qué condiciones van a continuar los esfuerzos que se han venido desarrollando, ahora en concordancia con el CMCT. En este sentido, es impostergable disponer de un sistema de seguimiento y evaluación. Para ello, la ETJ ofrece una oportunidad única, por los re- sultados que ya muestra como medición basal y por su solidez de diseño que la hacen adecuada como sistema de vigilancia epidemiológica.

\section{Agradecimientos}

Los autores agradecen a Fogarty International Center of the National Institutes of Health de Estados Unidos por el apoyo recibido a través del financiamiento No. R01-HL-73699. El primer autor agradece en especial a la Organización Panamericana de la Salud, oficina regional de la Organización Mundial de la Salud, que le otorgó una beca parcial para cursar los estudios de Maestría en Salud Pública. De igual manera, los autores reconocen la lectura exhaustiva de la versión final y los valiosos comentarios que aportó la doctora Cristina Rius Gibert.

\section{Referencias}

I. Peto R, Lopez AD. Future worldwide health effects of current smoking patterns. In: Koop CE, Pearson CE, Schwartz MR, eds. Critical Issues in Global Health. San Francisco, Calif.: Jossey-Bass 200 I;I54-I6I 2. The WHO Framework Convention on Tobacco Control, World Health Assembly Resolution 56. I, May 2I, 2003.

3. Global Tobacco Surveillance System Collaborating Group: Global Tobacco Surveillance System (GTSS): purpose, production, and potential. J Sch Health 2005;75(I): I5-24.

4. Global Youth Tobacco Survey Collaborating Group: differences in worlwide tobacco use by gender: findings from the GlobalYouth Tobacco Survey. J Sch Health 2003;73(6):207-2I 5.

5. Samet JM, Yoon SY, eds. Women and the tobacco epidemic: challenges for the 2 I st century. Geneva:The World Health Organization and The Institute for Global Tobacco Control, Johns Hopkins School of Public Health, 200I.

6.Women and Smoking: a report of the surgeon general. Rockville, Md: US Dept Health and Human Services, Public Health Services, Office of the Surgeon General, 200I.

7. Pierce JP, Choi WS, Gilpin EA, Farkas AJ, Merritt RK.Validation of susceptibility as a predictor of which adolescents take up smoking in the United States. Health Psychol 1996;15(5):355-36I.

8. Valdés-Salgado R. Resultados de la Encuesta Mundial para el Personal de las Escuelas aplicada en la Ciudad de México, en:Valdés-Salgado R, Meneses-González F, Lazcano-Ponce EC, Hernández-Ramos MI, Hernández-Avila M. Encuesta sobre Tabaquismo en Jóvenes, México 2003. Cuernavaca, Morelos: Instituto Nacional de Salud Pública, 2004; III-II8.

9. Wipfli H, Stillman F, Tamplin S, da Costa e Silva VL, Yach D, Samet JM. Achieving the Framework Convention on Tobacco Control's potential by investing in national capacity. Tob Control 2004; 13:433-437.

I0.Asma S, Warren W, Althomson S, Wisotzky M, Woollery T, Henson R. Addressing the Chronic Disease Burden with Tobacco Control

Programs. Public Health Rep 2004; I 19: 253-262.

I I. Stillman FA, Hartman AM, Graubard BI, Gilpin EA, Murray DM, Gibson JT. Evaluation of the American Stop Smoking Intervention Study (ASSIST): a report of outcomes. J Natl Cancer Inst 2003;95(22):I68I-I69I.

12. Warren W, Jones NR, Eriksen MP,Asma S, for the Global Tobacco Surveillance System Collaborating Group: patterns of global tobacco use in young people and implications for future chronic disease burden in adults. Lancet 2006;367:749-753. 